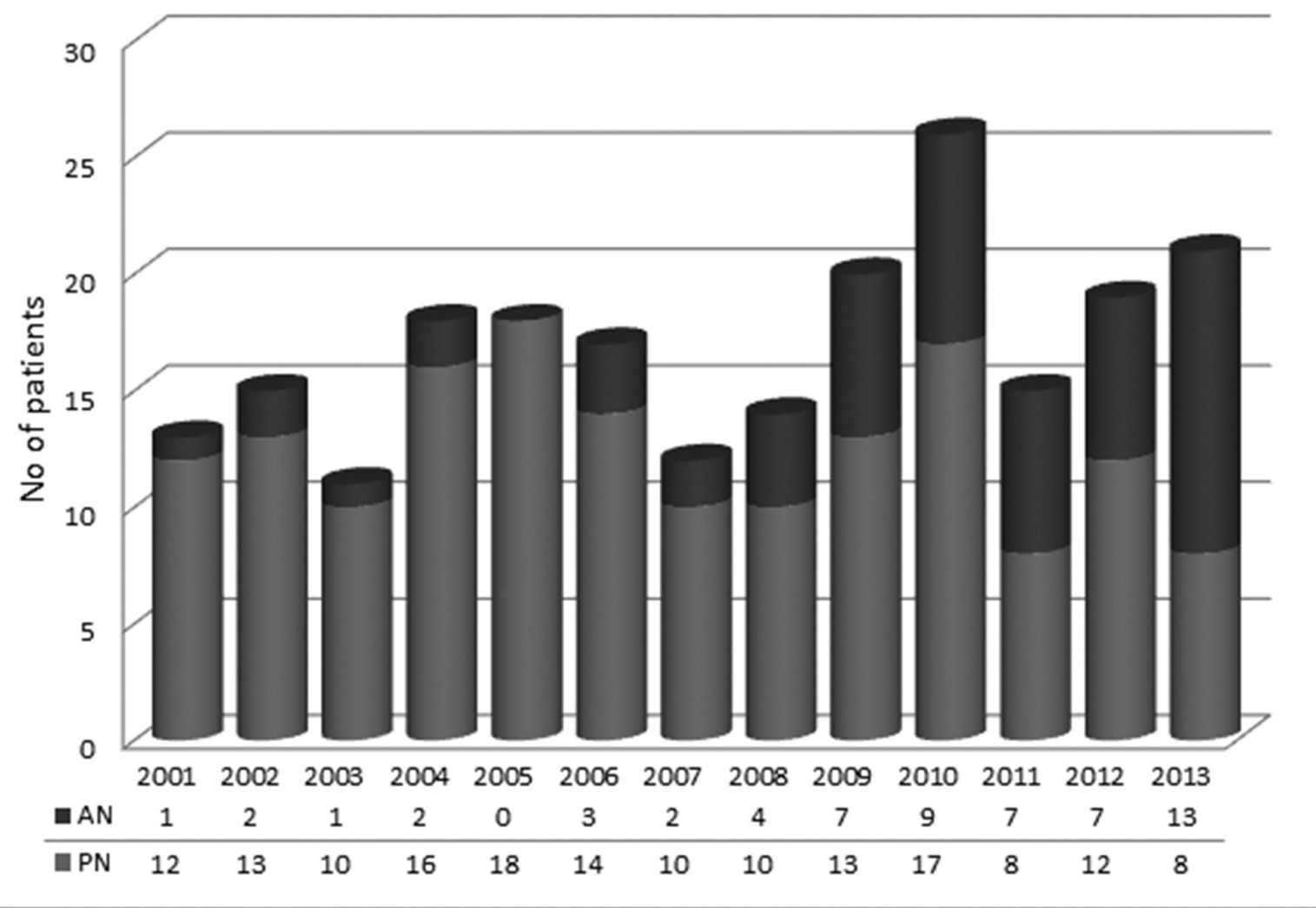

Abstract G162 Figure 1 Trends in timing of diagnosis for neonates with TGA + /- VSD undergoing the arterial switch procedure at our institution

\section{G163 A REVIEW OF THE CLINICAL PRACTICE OF PECS (PAEDIATRICIANS WITH EXPERTISE IN CARDIOLOGY) IN THE UK}

${ }^{1} Y$ Singh, ${ }^{2} \mathrm{H}$ Andrews. 'The Rosie Hospital, Cambridge University NHS Trust, Cambridge, UK; ${ }^{2}$ College of Medical and Dental Sciences, University of Birmingham Medical School, Birmingham, UK

\subsection{6/archdischild-2015-308599.159}

Aim To investigate which clinical diagnostic services (including echocardiography) are provided by PECs and to determine the extent and variability of the service support provided for paediatric cardiology in non-specialist district hospitals in the UK.

Methods A piloted, structured web-based questionnaire was sent to all contacts on the PECSIG and NICHe databases and to Consultants in the hospitals which were not represented within these databases via an NHS directory. Non-responders were followed up by telephone.

Results 177 hospitals were contacted and 141 responses were obtained (80\% response rate).

In total, $83 \%(117 / 141)$ of the responding non-specialist centres offered a paediatric echocardiography service. Within these hospitals, most of the echocardiography was performed by PECs (79\%, 92/117). Support for this service was offered by Consultant paediatricians and/or neonatologists in 30\% (35/117) of hospitals. Specialist technicians performed paediatric echocardiography in only $12 \%(14 / 117)$ of the hospitals where this service was available. Overall, 17\% (24/141) of hospitals did not offer paediatric echocardiography whatsoever.

Most hospitals offered other non-interventional paediatric cardiology adjunct diagnostics services including 12 lead ECG (96\%), Holter ECG (91\%) and 24-hour ambulatory blood pressure monitoring (74\%). Fewer provided long term external cardiac monitoring (54\%) and exercise testing (47\%).

Less than half of hospitals (48\%) had dedicated secretarial support for paediatric cardiology, only $14 \%$ had dedicated nursing staff and just $7 \%$ offered psychological support for patients and their families. Interestingly, $45 \%$ of hospitals had neither secretarial nor specialist nursing support for paediatric cardiology.

Some form of telemedicine, used for the purposes of paediatric cardiology, was available in 52\% (74/141) of hospitals. Where telemedicine was utilised, PACS was the most common form (24\%, 34/141).

Conclusion There remains some inconsistency in the provision of paediatric cardiology diagnostic services (e.g. echocardiography) at the district level. Development of telemedicine facilities in this field is likely to play an important role in making timely accurate diagnosis and management, and should be a focus for improvement in successive years.

\section{G164 ABSTRACT WITHDRAWN}

\section{G165 SIGNS OF DETERIORATION IN INFANTS DISCHARGED HOME FOLLOWING CONGENITAL HEART SURGERY IN THE FIRST YEAR OF LIFE: A QUALITATIVE STUDY}

1J Tregay, ' K Brown, ${ }^{2} \mathrm{~S}$ Crowe, ${ }^{1} \mathrm{C}$ Bull, ${ }^{3} \mathrm{RL}$ Knowles, 'J Wray. 'Great Ormond Street Hospital NHS Foundation Trust, London, UK; ${ }^{2}$ Clinical Operational Research Unit, University College London, London, UK; ${ }^{3}$ Institute of Child Health, University College London, London, UK

\subsection{6/archdischild-2015-308599.160}


Aims To describe the ways in which parents recognise and make decisions about their child's symptoms following discharge home after congenital heart interventions in the first year of life and their experiences of seeking help.

Methods This was a qualitative study involving semi-structured interviews with parents. Twenty-one parents were recruited to the study. Parents all had a child who had congenital heart surgery in their first year of life between September 2009 and October 2013 at one of three UK cardiac centres; the children had either died or were readmitted as an emergency following initial discharge.

Results Some parents were unable to identify any early warning signs at all. Others described symptoms of deterioration including: changes in feeding and appearance, respiratory distress, and subtle behavioural changes that are not routinely highlighted to parents at discharge. Several barriers to accessing prompt medical assistance were identified including parents feeling that their concerns were not taken seriously, and long wait times and lack of protocols at A\&E.

Conclusions Our study highlights behavioural symptoms as being a potentially under-emphasised sign of deterioration and identifies a number of barriers to parents accessing support when they are concerned. It is important that parents are encouraged to seek advice at the earliest opportunity and that those health professionals at the front line have access to the information they need in order to respond in an appropriate and timely way. A role for home monitoring was also noted as potentially useful in identifying at risk children who appear clinically well.

Funding National Institute for Health Research Health Services and Delivery Research programme (Project No: 10/2002/29) and the Health Foundation.

\section{G166 RISK OF DEATH OR EMERGENCY READMISSION FOLLOWING DISCHARGE AFTER INFANT CARDIAC INTERVENTION FOR CONGENITAL HEART DISEASE}

${ }^{1} S$ Crowe, ${ }^{2} \mathrm{D}$ Ridout, ${ }^{2} \mathrm{R}$ Knowles, ${ }^{3} \mathrm{~J}$ Tregay, ${ }^{3} \mathrm{~J}$ Wray, ${ }^{4} \mathrm{D}$ Barron, ${ }^{5} \mathrm{D}$ Cunningham, ${ }^{6} \mathrm{R}$ Parslow, ${ }^{7} \mathrm{R}$ Franklin, ${ }^{3} \mathrm{C}$ Bull, ${ }^{3} \mathrm{~K}$ Brown. ${ }^{1}$ Clinical Operational Research Unit, University College London, London, UK; ${ }^{2}$ Institute of Child Health, University College London, London, UK; ${ }^{3}$ Great Ormond Street Hospital NHS Foundation Trust, London, UK; ${ }^{4}$ Birmingham Children's Hospital NHS Foundation Trust, Birmingham, UK; ${ }^{5}$ National Institute for Cardiovascular Outcomes Research, University College London, London, UK; ${ }^{6}$ Paediatric Intensive Care Audit Network, University of Leeds, Leeds, UK; ${ }^{7}$ Royal Brompton and Harefield Hospitals NHS Foundation Trust, London, UK

\subsection{6/archdischild-2015-308599.161}

Aims 1) To identify risk factors for death or unplanned readmission within one year following hospital discharge after cardiac intervention for congenital heart disease. 2) To characterise patient groups at highest risk who would benefit from targeted intervention.

Methods Records in the national congenital cardiac surgical audit (NICOR) pertaining to UK infants who had a cardiac surgery or intervention aged under 12 months between 01/01/2005 and 31/12/2010 were matched with intensive care admission records in Paediatric Intensive Care Audit Network (PICANET); linked records with known life-status were obtained for 7634 infants. Outcome measures were: Outcome 1 - death within 1-year following discharge; Outcome 2 - Outcome 1 or emergency readmission to PICU within 1-year following discharge. Potential risk factors available from either dataset were pre-specified and univariate and multivariate logistic regression used to investigate the effects of these on each outcome. Classification and regression tree (CART) analysis was used to identify distinct patient groups differentiated by risk of Outcome 2, each defined by a set of patient characteristics.

Results 3.2\% (246/7643) and 6.7\% (514/7643) of infants experienced Outcome 1 and 2 respectively. Fitted multivariate models for both outcomes were robust in risk factor selection (Outcome 1 - ROC AUC $=0.78,95 \%$ CI $[0.75,0.82]$; Outcome 2 - ROC $\mathrm{AUC}=0.78[0.75,0.80])$. Risk factors significant in the multivariate Outcome 2 model were: age at procedure, weight $\mathrm{z}$ score, cardiac procedure, cardiac diagnosis, non-cardiac congenital anomaly, neurodevelopmental condition, prematurity $(<37$ weeks gestation), ethnicity, and length of stay in specialist centre (LOS). Clinical deterioration was additionally significant to Outcome 1 whilst neurodevelopmental condition and acquired diagnoses were not. Key defining characteristics of infants in the patient groups identified as higher risk were [\% Outcome 2]: (1) neurodevelopmental conditions [24\%]; (2) Hypoplastic left heart, single ventricle or pulmonary atresia [15\%]; (3) Congenital anomalies and LOS > 1 month [24\% risk]; (4) No congenital anomalies and LOS $>1$ month [9\% risk].

Conclusions Understanding patient risk groups should inform recommendations for improving services, support development of interventions to mitigate each profile of risk and facilitate evaluation of the priority and feasibility of targeting each group.

\section{G167(P) CHEST PAIN IN CHILDREN IS RARELY CARDIAC. DOES ECG HELP?}

P Babu, S Dickson, D Dhar, D Hapuarachchi, K Murtagh, F Damda. Department of Paediatrics, University Hospital Lewisham, Lewisham and Greenwich NHS Trust, Lewisham, UK

\subsection{6/archdischild-2015-308599.162}

Aims Chest pain is a common reason for presentation to the children's emergency department (ED). It is known that chest pain in children, compared to adults, is much less likely to be caused by cardiovascular disease. Electrocardiographs (ECGs) are cheap, fast and readily available. When interpreted appropriately they can be useful in demonstrating cardiac causes of chest pain. We aimed firstly to determine the incidence and likely causes of paediatric chest pain presenting to our busy Children's ED and secondly to analyse the usefulness of ECGs in this cohort.

Methods We retrospectively analysed the ED case notes of all children (aged under 16 years) presenting with chest pain over a 4 year period (April 2009 to March 2013) to a busy Children's ED in an urban district general hospital.

Results 1126 attendances presented with chest pain, approximately $1 \%$ of all attendances over four years. Of those with chest pain 54\% were male and the modal age of presentation was 12 years. Based on history and clinical examination the commonest cause attributed to chest pain was musculoskeletal. Only $1 \%$ of cases had a possible cardiac aetiology; 1 patient had pericarditis, 1 patient had myocarditis, 2 patients had a pre-existing cardiac condition, 3 patients had arrhythmias and 8 were under investigation for recurrent palpitations. Thirty per cent of patients with chest pain had an ECG carried out. Patients with cardiovascular, psychiatric and musculoskeletal diagnoses were most likely to have had an ECG done. The majority of ECGs were normal (91\%). The commonest abnormality was high take off/mild ST elevation, with only $10 \%$ of such patients having cardiac enzymes requested. Some of the ECG abnormalities identified could not be attributed to chest pain. 